\title{
Detection of hot gas in the filament connecting the clusters of galaxies Abell 222 and Abell 223
}

\author{
N. Werner ${ }^{1}$, A. Finoguenov ${ }^{2}$, J. S. Kaastra ${ }^{1,3}$, A. Simionescu ${ }^{2}$, J. P. Dietrich ${ }^{4}$, J. Vink ${ }^{3}$, and H. Böhringer ${ }^{2}$ \\ 1 SRON Netherlands Institute for Space Research, Sorbonnelaan 2, 3584 CA Utrecht, The Netherlands \\ e-mail: n.werner@sron.nl \\ 2 Max-Planck-Institut für Extraterrestrische Physik, 85748 Garching, Germany \\ 3 Astronomical Institute, Utrecht University, PO Box 80000, 3508 TA Utrecht, The Netherlands \\ ${ }^{4}$ ESO, Karl-Schwarzschild-Str. 2, 85748 Garching, Germany
}

Received 18 February 2008 / Accepted 17 March 2008

\section{ABSTRACT}

\begin{abstract}
Context. About half of the baryons in the local Universe are invisible and - according to simulations - their dominant fraction resides in filaments connecting clusters of galaxies in the form of low density gas with temperatures in the range of $10^{5}<T<10^{7} \mathrm{~K}$. This warm-hot intergalactic medium has never been detected indisputably using X-ray observations.

Aims. We aim to probe the low gas densities expected in the large-scale structure filaments by observing a filament connecting the massive clusters of galaxies A 222 and A $223(z=0.21)$, which has a favorable orientation approximately along our line-of-sight. This filament has been previously detected using weak lensing data and as an over-density of colour-selected galaxies. Methods. We analyse X-ray images and spectra obtained from a deep observation (144 ks) of A 222/223 with XMM-Newton.

Results. We present observational evidence of X-ray emission from the filament connecting the two clusters. We detect the filament in the wavelet-decomposed soft-band $(0.5-2.0 \mathrm{keV}) \mathrm{X}$-ray image with a $5 \sigma$ significance. Following the emission down to the $3 \sigma$ significance level, the observed filament is $\approx 1.2 \mathrm{Mpc}$ wide. The temperature of the gas associated with the filament, determined from the spectra, is $k T=0.91 \pm 0.25 \mathrm{keV}$, and its emission measure corresponds to a baryon density of $(3.4 \pm 1.3) \times 10^{-5}(l / 15 \mathrm{Mpc})^{-1 / 2} \mathrm{~cm}^{-3}$, where $l$ is the length of the filament along the line-of-sight. This density corresponds to a baryon over-density of $\rho /\left\langle\rho_{\mathrm{C}}\right\rangle \approx 150$. The properties of the gas in the filament are consistent with results of simulations of the densest and hottest parts of the warm-hot intergalactic medium.
\end{abstract}

Key words. cosmology: large-scale structure of Universe - X-rays: galaxies: clusters galaxies: clusters: individual: Abell 222 - galaxies: clusters: individual: Abell 223

\section{Introduction}

According to the standard theory of structure formation, the spatial distribution of matter in the Universe evolved from small perturbations in the primordial density field into a complex structure of sheets and filaments with clusters of galaxies at the intersections of this filamentary structure. The filaments have been identified in optical surveys of galaxies (e.g. Joeveer et al. 1978; Baugh et al. 2004; Tegmark et al. 2004), but the dominant fraction of their baryons is probably in the form of a low density warm-hot gas emitting predominantly soft X-rays.

The existence of this warm-hot intergalactic medium (WHIM) permeating the cosmic-web is predicted by numerical hydrodynamic simulations (Cen \& Ostriker 1999; Davé et al. $2001)$, which show that in the present epoch $(z \lesssim 1-2)$ about $30 \%$ to $40 \%$ of the total baryonic matter resides in the filaments connecting clusters of galaxies. These simulations predict that the WHIM spans a temperature range of $10^{5}<T<10^{7} \mathrm{~K}$ and a density range of $4 \times 10^{-6} \mathrm{~cm}^{-3}$ to $10^{-4} \mathrm{~cm}^{-3}$, which corresponds to 15-400 times the mean baryonic density of the Universe. Gas at these temperatures and densities is difficult to detect (Davé et al. 2001).

Several attempts have been made to detect the WHIM both in absorption and emission, but none have succeeded in providing an unambiguous detection. Marginal detection of O VIII and Ne IX absorption features in XMM-Newton RGS spectra of distant quasars was reported in the vicinity of the Virgo and Coma clusters and interpreted as indication of the presence of dense and hot WHIM concentrations near the clusters (Fujimoto et al. 2004; Takei et al. 2007). A blind search for WHIM was performed by Nicastro et al. (2005), who reported the discovery of two intervening systems at $z>0$ in absorption along the line-of-sight to the blazar Mrk 421 in the Chandra Low Energy Transmission Grating Spectrometer (LETGS) spectra. However, Kaastra et al. (2006) carefully reanalysed the LETGS spectra, confirmed the apparent strength of several features, but showed that their detection was not statistically significant. Furthermore, the results reported by Nicastro et al. (2005) were neither confirmed by later Chandra LETGS observations nor by the XMM-Newton RGS data of Mrk 421 (Kaastra et al. 2006; Rasmussen et al. 2007). The detection of the WHIM in absorption using current instrumentation will remain difficult, because the expected absorption features are weak. In the large searched redshift space to distant blazars, statistical fluctuations with a strength comparable to the strength of absorption lines expected from the WHIM will therefore always appear. A search for the WHIM from the densest and hottest parts of the large-scale structure filaments with current instruments is more realistic in emission than in absorption.

Searches in emission with ROSAT and XMM-Newton have found several candidates for WHIM filaments (Kull \& Böhringer 1999; Zappacosta et al. 2002; Finoguenov et al. 2003; 
Kaastra et al. 2003). However, the association of the detected soft emission with the WHIM has always been difficult and uncertain. In some cases, there is still a possible confusion with the Galactic foreground emission, or with the Solar-wind charge exchange emission that also produces $\mathrm{O}$ VII lines and soft emission in excess of cluster emission.

Since the X-ray emissivity scales with the square of the gas density, X-ray emission studies are most sensitive to the densest gas concentrations of the WHIM that are located, according to simulations, near clusters of galaxies. The most appropriate method to study the physical properties of the WHIM is therefore to target distant binary clusters in which the connecting filament bridge is aligned approximately along our lineof-sight. A filament between cluster pairs observed with such a favourable geometry has a higher surface brightness than filaments observed at a different orientation angle. By observing the variation of the surface brightness in X-ray images extracted in the low energy band, the presence of a WHIM filament can be revealed as a bridge between the clusters. This is a differential test with respect to the background and it is less sensitive to systematic uncertainties.

The most promising target for detecting the warm-hot gas in a filament is Abell 222/223, a close pair of massive clusters of galaxies at redshift $z \approx 0.21$, separated by $\sim 14^{\prime}$ on the sky, which corresponds to a projected distance of $\sim 2.8 \mathrm{Mpc}$. Assuming that both clusters are part of the Hubble flow without peculiar velocities, the observed redshift difference of $\Delta z=0.005 \pm 0.001$ (Dietrich et al. 2002) translates to a physical separation along the line of sight of $15 \pm 3 \mathrm{Mpc}$. Dietrich et al. (2005) reported indications of a filament connecting both clusters using weak lensing, optical, and X-ray (ROSAT) data. They found an X-ray bridge with a linear extent of about $1.3 \mathrm{Mpc}$ in projection, connecting the clusters at a $5 \sigma$ significance level, and an over-density of galaxies in the filament at a $7 \sigma$ significance level. In the weak lensing image, the filament was present at a $2 \sigma$ significance.

We present results of a new, deep (144 ks) XMM-Newton observation of the bridge connecting the two clusters. We confirm its presence by both imaging and spectroscopy, and we estimate the temperature, density, entropy, and the total mass of the gas in the filament. Throughout the paper we use $H_{0}=$ $70 \mathrm{~km} \mathrm{~s}^{-1} \mathrm{Mpc}^{-1}, \Omega_{M}=0.3, \Omega_{\Lambda}=0.7$, which imply a linear scale of $206 \mathrm{kpc} \operatorname{arcmin}^{-1}$ at the redshift of $z=0.21$. Unless specified otherwise, all errors are at the $68 \%$ confidence level for one interesting parameter $\left(\Delta \chi^{2}=1\right)$.

\section{Observations and data analysis}

The pair of clusters Abell 222/223 was observed with XMM-Newton in two very nearby pointings centred on the filament on June 18 and 22, 2007 (revolutions 1378 and 1380) with a total exposure time of $144 \mathrm{ks}$. The calibrated event files were produced using the 7.1.0 version of the XMM-Newton Science Analysis System (SAS). After removing the time intervals affected by high particle-background, we obtained $62.6 \mathrm{ks}$ of good time for EPIC/MOS and $34.4 \mathrm{ks}$ for EPIC/pn.

\subsection{Image analysis}

We coadded the final $0.5-2.0 \mathrm{keV}$ and $2.0-7.5 \mathrm{keV}$ images from all instruments and both pointings. The background was subtracted and the images were corrected for vignetting. The chip number 4 in EPIC/MOS1 was excluded from the analysis because of the anomalously high flux in the soft band (for more
Table 1. The CXB components determined in a region free of clusters emission outside of the virial radii of the clusters. The unabsorbed fluxes are determined in the $0.3-10.0 \mathrm{keV}$ band. $f$ indicates parameters kept fixed during spectral fitting.

\begin{tabular}{lcc}
\hline \hline Comp. & $\begin{array}{c}k T / \Gamma \\
(\mathrm{keV} / \text { phot. ind. })\end{array}$ & $\begin{array}{c}\text { Flux } \\
\left(10^{-12} \mathrm{erg} \mathrm{cm}^{-2} \mathrm{~s}^{-1} \mathrm{deg}^{-2}\right)\end{array}$ \\
\hline LHB & $k T=0.08^{f}$ & $3.4 \pm 1.1$ \\
GH & $k T=0.17 \pm 0.03$ & $2.9 \pm 0.9$ \\
EPL & $\Gamma=1.41^{f}$ & $22^{f}$ \\
PL & $\Gamma=0.78 \pm 0.16$ & $49 \pm 7$ \\
\hline
\end{tabular}

details see Snowden et al. 2008). To eliminate the flux pollution from the faint point sources due to the large wings of the point-spread function (PSF) of XMM-Newton, we applied an image-restoration technique (Finoguenov et al., in prep) that uses a symmetric model for the PSF of XMM-Newton calibrated by Ghizzardi (2001). The flux from the extended wings of the PSF of sources detected on small scales (sum of the $8^{\prime \prime}$ and $16^{\prime \prime}$ scale) was estimated by performing a scale-wise wavelet analysis (Vikhlinin et al. 1998). This estimated flux was used to subtract the PSF model prediction from the larger scales and to accordingly increase the flux on the smaller scales. The uncertainties in the point-source subtraction were added to the total error budget to reduce the significance of the residuals.

\subsection{Spectral analysis}

We extracted the filament spectrum from a circular extraction region with a radius of $1.6^{\prime}$, located between the clusters, and centred at a projected distance of $5.76^{\prime}(1.19 \mathrm{Mpc})$ from both cluster cores. We determined the background spectrum using two independent sets of regions to provide a consistency check. We used, firstly, a circular region of $3.2^{\prime}$ radius, located at a projected distance larger than $1.6 \mathrm{Mpc}$ from both clusters, which is beyond their virial radii determined using weak lensing analysis $\left(r_{200}=1.28 \pm 0.11 \mathrm{Mpc}\right.$ for A 222 and $r_{200}=1.55 \pm 0.15 \mathrm{Mpc}$ for A 223; Dietrich et al. 2005). Secondly, we extracted two other circles of $1.6^{\prime}$ radii located on the same central chip of the EPIC/MOS detectors. Both circles are located at the same projected distance to one of the clusters as the extraction region centred on the filament. Using the same chip and the same projected distance to the clusters for determining both the source and the background spectra ensures the best possible subtraction of the instrumental and X-ray background components. Point sources were excluded from the spectral extraction regions. For the identification of point sources, we also used archival Chandra data $(45.7 \mathrm{ks})$. The regions used for spectral extraction, for both the filament and the background, are indicated in Fig. 1.

We subtracted the instrumental background from all spectra using closed-filter observations. Since the instrumental background level changes from observation to observation and increases with time over the years, we normalised the closed-filter data, for each extraction region, by the $10-12 \mathrm{keV}$ count rate for EPIC/MOS data and by the 10-14 keV count rate for EPIC/pn data. We then modelled the spectra for each background region set with the components described below, and used these models to subtract the cosmic X-ray background from the filament spectrum.

We analysed the spectra using the SPEX package (Kaastra et al. 1996) in the 0.35 to $7 \mathrm{keV}$ band for EPIC/MOS and 0.6-7 keV band for EPIC/pn, ignoring the 1.3-1.8 keV band because of the presence of strong instrumental lines. We fixed the Galactic absorption in our model to the value determined from 


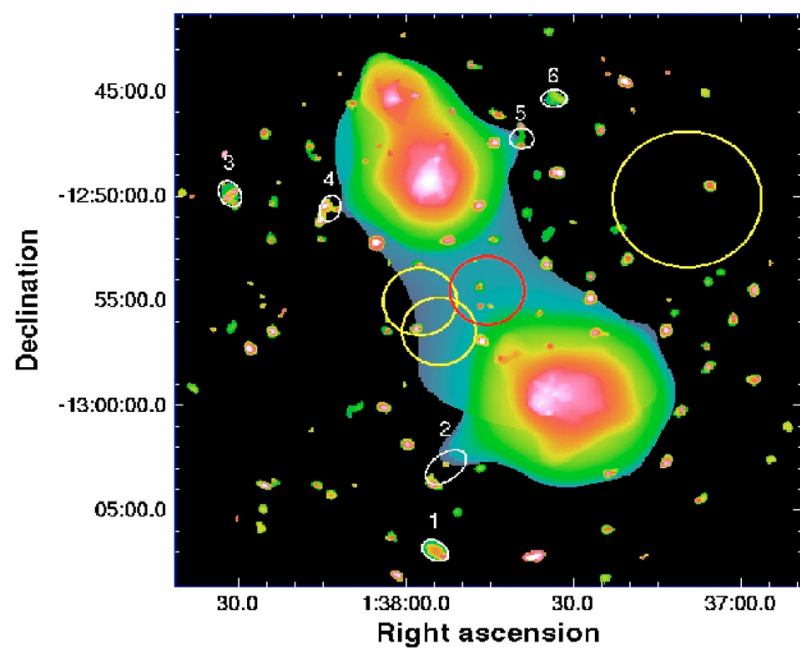

Fig. 1. Wavelet-decomposed 0.5-2.0 keV image of Abell 222 (to the South) and Abell 223 (the two X-ray peaks to the North). We show only sources with $>5 \sigma$ detection, but for these sources we follow the emission down to $3 \sigma$. The filament connecting the two massive clusters is clearly visible in the image. The regions used to extract the filament spectrum and to determine the background parameters are indicated by red and yellow circles, respectively.

H I radio data $\left(N_{\mathrm{H}}=1.6 \times 10^{20} \mathrm{~cm}^{-2}\right.$, Kalberla et al. 2005). We modelled the background with four components: the extragalactic power-law (EPL), to account for the integrated emission of unresolved point sources (assuming a photon index $\Gamma=1.41$ and a $0.3-10.0 \mathrm{keV}$ flux of $2.2 \times 10^{-11} \mathrm{erg} \mathrm{s}^{-1} \mathrm{~cm}^{-2} \mathrm{deg}^{-2}$ after extraction of point sources, De Luca \& Molendi 2004); two thermal components, to account for the local hot bubble (LHB) emission $\left(k T_{1}=0.08 \mathrm{keV}\right)$ and for the Galactic halo $(\mathrm{GH})$ emission $\left(k T_{2} \sim 0.2 \mathrm{keV}\right)$; and an additional power-law to account for the contamination from the residual soft proton particle background. The best-fitted fluxes, temperatures, and photon indices of the background components, using the $3.2^{\prime}$ circular extraction region, are shown in Table 1.

We modelled the spectrum of the filament with a collisionally-ionized plasma model (MEKAL), the metallicity of which was set to 0.2 Solar (proto-Solar abundances by Lodders 2003), which is the lowest value found in the outskirts of clusters and groups (Fujita et al. 2008; Buote et al. 2004). However, since the average metallicity of the WHIM is unknown, we report results also for metallicities of $0.0,0.1$, and 0.3 Solar. The free parameters in the fitted model are the temperature and the emission measure of the plasma.

\section{Results}

\subsection{Imaging}

We detect the filament in the wavelet-decomposed soft-band $(0.5-2.0 \mathrm{keV}) \mathrm{X}$-ray image with $5 \sigma$ significance. In Fig. 1, we show the wavelet-decomposed image produced by setting the detection threshold to $5 \sigma$ and following the emission down to $3 \sigma$ (for details of the wavelet decomposition technique see Vikhlinin et al. 1998). There is a clear bridge in the soft emission between the clusters, which originates in an extended component. We note that no such features are detected in any XMM-Newton mosaics of empty fields in observations of similar but also much larger depths. We do not detect the bridge between the clusters in the $2.0-7.5 \mathrm{keV}$ image.

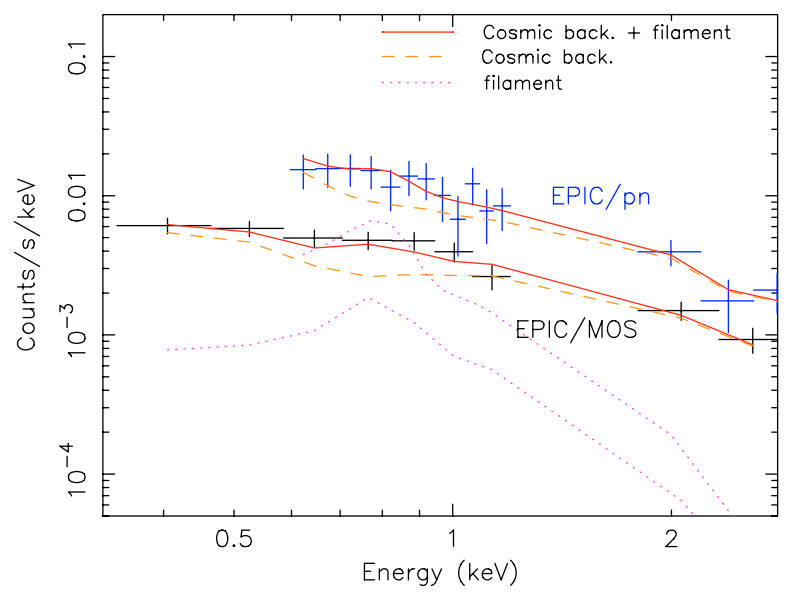

Fig. 2. The spectrum of the filament between the clusters A 222/223 - the data points on the top were obtained by EPIC/pn and below by EPIC/MOS. The contributions from the X-ray background and from the filament to the total model are shown separately.

To verify our result, we created an image modelling the $\mathrm{X}$-ray emission from the two clusters without a filament with two beta models determined by fitting the surface-brightness profile of A 222 and A 223. We then applied the wavelet-decomposition algorithm to this image, which did not reveal a bridge such as that observed between A 222 and A 223.

If the emission in the bridge between the clusters originated in a group of galaxies within the filament, then this group would be well resolved in the image. Groups of galaxies at the redshift of this cluster emit on spatial scales of $\approx 0.5^{\prime}$ as shown in Fig. 1 by the six extended sources detected at the $4 \sigma$ significance around Abell 222/223 marked by ellipses numbered from 1 to 6 . The filament seen in the image is about $6^{\prime}$ wide, which at the redshift of the cluster corresponds to about 1.2 Mpc.

The observed filament is about an order of magnitude fainter than the ROSAT PSPC detected filament reported by Dietrich et al. (2005). The ROSAT detection was based on an image smoothed by a Gaussian with $\sigma=1.75^{\prime}$, and the filament could be the result of the combination of emission from the cluster outskirts and from a few previously unresolved point-sources between the clusters.

The point-sources (mostly AGNs) detected by XMM-Newton and Chandra do not show an overdensity between the clusters, and the uniform contribution from the distant unresolved AGNs was subtracted from our image. Our detection limit for point sources is $\sim 0.8 \times 10^{-15} \mathrm{erg} \mathrm{s}^{-1} \mathrm{~cm}^{-2}$, which corresponds to a luminosity of $10^{41} \mathrm{erg} \mathrm{s}^{-1}$ for the faintest resolved galaxy at the cluster redshift. The number of X-ray emitting galaxies with this luminosity required to account for the observed emission in the filament would be $\sim 10$ times larger than expected from the distribution function of Hasinger (1998), assuming a filament over-density of $\rho /\langle\rho\rangle \sim 100$.

\subsection{Spectroscopy}

The clusters of galaxies Abell 222 and Abell 223 are bright and relatively hot. The temperature of A 222, extracted from a region with a radius of $2^{\prime}$ is $4.43 \pm 0.11 \mathrm{keV}$ and the temperature of southern core of A 223 extracted within the same radius is $5.31 \pm$ $0.10 \mathrm{keV}$. However, we focus here on the bridge connecting the two clusters. 
As shown in Fig. 2, the spectrum extracted from the filament shows, between $\sim 0.5$ and $\sim 1.0 \mathrm{keV}, \sim 30 \%$ emission in excess of the background model, which was determined outside the cluster virial radii (see Table 1). We fit this excess emission with a thermal model with a temperature of $k T=0.91 \pm 0.24 \pm 0.07 \mathrm{keV}$, where the first error is the statistical error and the second error is due to the uncertainty in the background level, which was determined by varying the background components in the spectral fit within their $1 \sigma$ errors. The best-fit emission measure of the filament, which is defined to be $E M=\int n_{\mathrm{H}} n_{\mathrm{e}} \mathrm{d} V$, where $n_{\mathrm{H}}$ and $n_{\mathrm{e}}$ are the proton and electron number densities and $V$ is the volume of the emitting region, is $(1.72 \pm 0.50 \pm 0.45) \times 10^{65} \mathrm{~cm}^{-3}$. Assuming that both errors have a Gaussian distribution, the emission measure of the plasma in the filament within our extraction region is $E M=(1.72 \pm 0.67) \times 10^{65} \mathrm{~cm}^{-3}(2.6 \sigma)$. Fitting individually EPIC/MOS and EPIC/pn the best-fit temperatures are $0.94 \pm 0.23 \mathrm{keV}$ and $0.85 \pm 0.30 \mathrm{keV}$, respectively, and the statistical significance of the excess emission is $3.5 \sigma$ and $1.8 \sigma$. The derived emission measure depends on the assumed metallicity. For a higher metallicity, we obtain a lower emission measure (for $Z=0.3$ Solar, $E M=1.3 \times 10^{65} \mathrm{~cm}^{-3}$ ), while for a lower metallicity the emission measure is higher (for $Z=0.1$ Solar, $E M=2.4 \times 10^{65} \mathrm{~cm}^{-3}$ and for $Z=0$ Solar, $E M=4.13 \times 10^{65} \mathrm{~cm}^{-3}$ ). The plasma temperature does not change significantly as a function of the assumed metallicity. The $0.3-10.0 \mathrm{keV}$ luminosity of the filament, within our extraction region, is $1.4 \times 10^{42} \mathrm{erg} \mathrm{s}^{-1}$. In the COSMOS survey, a number of groups have been detected at these luminosities and redshifts, but their typical spatial extent $\left(r_{500} \sim 1.3^{\prime}\right.$, Finoguenov et al., in prep.) is much smaller than that of this filament.

By determining the background properties using the two extraction regions at the central chip of EPIC/MOS and fitting only the EPIC/MOS data, we obtain a best-fit emission measure and temperature for the filament of $E M=(1.7 \pm 0.6) \times 10^{65} \mathrm{~cm}^{-3}$ and $k T=0.80 \pm 0.19 \mathrm{keV}$. These values are consistent with those obtained using a background region located at a larger distance on a different part of the detector. This minimizes chip-to-chip variations in the instrumental noise and allows to include some residual cluster emission in the form of an increased best-fit flux of the power-law model component. However, part of this increased power-law flux could be due to emission from the filament in the background regions, resulting in an oversubtraction of the filament.

\section{Discussion}

We detect X-ray emission from a bridge connecting the clusters A 222 and A 223. The temperature of the gas associated with this bridge is $k T=0.91 \pm 0.25 \mathrm{keV}$. If this gas is the intra-cluster medium (ICM) at the cluster outskirts, then assuming an emitting volume with a line-of-sight depth of $2.5 \mathrm{Mpc}$, our best-fit emission measure corresponds to a density of $\sim 1 \times 10^{-4} \mathrm{~cm}^{-3}$ and the entropy of the gas is $s=k T / n^{2 / 3} \sim 420 \mathrm{keV} \mathrm{cm}{ }^{2}$. Because of heating by accretion shocks, the cluster entropies rise towards the outskirts to values higher than $1000 \mathrm{keV} \mathrm{cm}^{2}$ outside $0.5 R_{200}$ (Pratt et al. 2006), and the entropy is expected to rise further out to the virial radius. The low entropy of the bridge implies that the emitting gas is in a filament that has not yet been reached by the shock-heating operating on the ICM in the cluster outskirts.

If the redshift difference between the clusters is only due to the Hubble flow, their line-of-sight distance difference is $15 \pm$ $3 \mathrm{Mpc}$. Assuming that the observed emission originates in hot gas within the filament connecting the clusters, the mean baryon density of the gas is $3.4 \times 10^{-5}(l / 15 \mathrm{Mpc})^{-1 / 2} \mathrm{~cm}^{-3}$, where $l$ is the length of the filament along the line-of-sight, which corresponds to 150 times the mean baryon density of the Universe. From this density, assuming $l=15 \mathrm{Mpc}$, and the temperature of $k T=$ $0.91 \mathrm{keV}$, we obtain an entropy $s=k T / n^{2 / 3} \sim 870 \mathrm{keV} \mathrm{cm}$. For a filament with a cylindrical shape, which is $1.2 \mathrm{Mpc}$ wide as indicated by the images, and assuming a constant density, we obtain a baryon mass of $1.8 \times 10^{13}(l / 15 \mathrm{Mpc})^{1 / 2} M_{\odot}$. Assuming a baryon fraction of 0.16 , the total mass of the filament is $\approx$ $1.1 \times 10^{14} M_{\odot}$.

The inferred properties of the gas in the filament are remarkably consistent with the results of the simulations by Dolag et al. (2006). They predict that within a distance of $\approx 15 \mathrm{Mpc}$ from a massive cluster, filaments connecting clusters have a baryon over-density of $\rho /\left\langle\rho_{\mathrm{C}}\right\rangle>100$ inside a radius of $\approx 1 \mathrm{Mpc}$ from the centre of the filament. They also found that the temperature of the gas along the bridges connecting some nearby clusters can be around $10^{7} \mathrm{~K}$, which is the temperature of the gas that we see between A 222 and A 223. The predicted X-ray surface brightness of filaments according to Dolag et al. (2006) is at most $10^{-16} \mathrm{erg} \mathrm{s}^{-1} \mathrm{~cm}^{-2} \operatorname{arcmin}^{-2}$. However, this predicted surface brightness was derived for zero metallicity (for a metallicity of $Z=0.2$ Solar, the predicted X-ray flux would increase, due to line emission, by a factor of $\sim 1.6$ ) and for the case of looking at the filament edge on. Line emission and a favourable geometry could increase the surface brightness to our observed value of $10^{-15} \mathrm{erg} \mathrm{s}^{-1} \mathrm{~cm}^{-2} \operatorname{arcmin}^{-2}$.

If the observed redshift difference is entirely due to peculiar velocities, $\Delta z=0.005$ would correspond to $\Delta v_{\mathrm{r}}=1500 \mathrm{~km} \mathrm{~s}^{-1}$, and both clusters would be at the same distance, in which case the virial radii of the clusters would partially overlap and they would be at the onset of a merger. However, if this is the case, then we would not expect to observe such a large amount of gas at these low temperatures between the clusters. Most of the mass from a previously present filament would have been accreted by the clusters and the gas in the interaction region would have been compressed and shock-heated to temperatures significantly higher than those observed. We therefore conclude that the redshift difference cannot be attributed entirely to peculiar velocities.

The temperature and average density of the observed gas associated with the filament indicates that we are detecting the hottest and densest phase of the WHIM. It is only detectable because of the favourable geometry of the massive filament connecting two large clusters of galaxies. According to simulations (e.g. Davé et al. 2001), however, the dominant fraction of the WHIM resides in a lower temperature, lower density phase, and its existence still remains to be proven observationally. The detection of the dominant fraction of the WHIM will be possible only using dedicated future instrumentation (e.g. see Paerels et al. 2008).

To further investigate the physical properties of the densest and hottest gas phase permeating the cosmic web, the observed sample of cluster pairs for which the large-scale structure filaments can be observed along our line-of-sight needs to be enlarged by other promising systems.

Acknowledgements. A.F. acknowledges support from BMBF/DLR under grant 50 OR 0207 and MPG. This work is based on observations obtained with XMM-Newton, an ESA science mission with instruments and contributions directly funded by ESA member states and the USA (NASA). The Netherlands Institute for Space Research (SRON) is supported financially by NWO, the Netherlands Organization for Scientific Research. 


\section{References}

Baugh, C. M., Croton, D. J., Gaztañaga, E., et al. 2004, MNRAS, 351, L44 Buote, D. A., Brighenti, F., \& Mathews, W. G. 2004, ApJ, 607, L91

Cen, R. \& Ostriker, J. P. 1999, ApJ, 514, 1

Davé, R., Cen, R., Ostriker, J. P., et al. 2001, ApJ, 552, 473

De Luca, A., \& Molendi, S. 2004, A\&A, 419, 837

Dietrich, J. P., Clowe, D. I., \& Soucail, G. 2002, A\&A, 394, 395

Dietrich, J. P., Schneider, P., Clowe, D., Romano-Díaz, E., \& Kerp, J. 2005, A\&A, 440, 453

Dolag, K., Meneghetti, M., Moscardini, L., Rasia, E., \& Bonaldi, A. 2006, MNRAS, 370, 656

Finoguenov, A., Briel, U. G., \& Henry, J. P. 2003, A\&A, 410, 777

Fujimoto, R., Takei, Y., Tamura, T., et al. 2004, PASJ, 56, L29

Fujita, Y., Tawa, N., Hayashida, K., et al. 2008, PASJ, 60, 343

Ghizzardi, S. 2001,

http://xmm.vilspa.esa.es/docs/documents/CAL-TN-0022-0.ps.gz

Hasinger, G. 1998, Astron. Nachr., 319, 37

Joeveer, M., Einasto, J., \& Tago, E. 1978, MNRAS, 185, 357

Kaastra, J. S., Mewe, R., \& Nieuwenhuijzen, H. 1996, in UV and X-ray Spectroscopy of Astrophysical and Laboratory Plasmas, K. Yamashita, \& T. Watanabe (Tokyo: Universal Academy Press), 411
Kaastra, J. S., Lieu, R., Tamura, T., Paerels, F. B. S., \& den Herder, J. W. 2003, A\&A, 397, 445

Kaastra, J. S., Werner, N., Herder, J. W. A. d., et al. 2006, ApJ, 652, 189

Kalberla, P. M. W., Burton, W. B., Hartmann, D., et al. 2005, A\&A, 440, 775

Kull, A. \& Böhringer, H. 1999, A\&A, 341, 23

Lodders, K. 2003, ApJ, 591, 1220

Nicastro, F., Mathur, S., Elvis, M., et al. 2005, ApJ, 629, 700

Paerels, F., Kaastra, J. S., Ohashi, T., et al. 2008, SSR, in press, [arXiv:0801.1064]

Pratt, G. W., Arnaud, M., \& Pointecouteau, E. 2006, A\&A, 446, 429

Rasmussen, A. P., Kahn, S. M., Paerels, F., et al. 2007, ApJ, 656, 129

Snowden, S. L., Mushotzky, R. F., Kuntz, K. D., \& Davis, D. S. 2008, A\&A, 478,615

Takei, Y., Henry, J. P., Finoguenov, A., et al. 2007, ApJ, 655, 831

Tegmark, M., Blanton, M. R., Strauss, M. A., et al. 2004, ApJ, 606, 702

Vikhlinin, A., McNamara, B. R., Forman, W., et al. 1998, ApJ, 502, 558

Zappacosta, L., Mannucci, F., Maiolino, R., et al. 2002, A\&A, 394, 7 